\title{
BRANDSTÄTTER, Klaus, Ratsfamilien und Tagelöhner. Die Bewohner von Hall in Tirol im ausgehenden Mittelalter
}

Julien Demade

\section{OpenEdition}

Édition électronique

URL : http://journals.openedition.org/ifha/1092

DOI : 10.4000/ifha.1092

ISSN : 2198-8943

\section{Éditeur}

IFRA - Institut franco-allemand (sciences historiques et sociales)

Référence électronique

Julien Demade, «BRANDSTÄTTER, Klaus, Ratsfamilien und Tagelöhner. Die Bewohner von Hall in Tirol im ausgehenden Mittelalter », Revue de l'IFHA [En ligne], Date de recension, mis en ligne le 01 janvier 2003, consulté le 22 septembre 2020. URL : http://journals.openedition.org/ifha/1092 ; DOI : https://doi.org/ 10.4000/ifha.1092

Ce document a été généré automatiquement le 22 septembre 2020.

(CIFHA 


\title{
BRANDSTÄTTER, Klaus, Ratsfamilien und Tagelöhner. Die Bewohner von Hall in Tirol im ausgehenden Mittelalter
}

\author{
Julien Demade
}

1 Tout l'intérêt de l'habilitation de K.B. vient de son objet, largement négligé par l'historiographie (si l'on excepte, du moins, ces dernières années) : une petite ville, en l'occurrence la tyrolienne Hall, près d'Innsbruck. Comme Hall, tout particulièrement en raison de la présence de mines de sel (attestées depuis 1232), de foires (depuis 1356) et d'un important atelier monétaire (depuis 1477), a déjà été, pour une localité d'environ 2 000 âmes au XVe s., l'objet de travaux scientifiques particulièrement nombreux, l'intérêt du livre de K.B. ne vient pas de ce qu'il comblerait une lacune de la Landesgeschichte tyrolienne, mais de la problématique qu'il utilise, et des résultats auxquels il aboutit. La problématique, centrée sur l'étude de la stratification sociale telle qu'Erich Maschke lui a donné (dans l'historiographie germanophone) ses lettres de noblesse, n'est certes pas neuve - mais en quoi cela serait-il preuve de son inintérêt ? Surtout, K.B., malgré l'absence de sources fiscales (sur lesquelles s'appuient presque exclusivement les études de la stratification sociale), parvient à mettre au jour des écarts extrêmes au sein de cette petite société urbaine, dont certains des membres s'assurent une richesse d'échelle supra-régionale grâce à leur participation aux bénéfices des différentes activités extractives (les salines de Hall d'abord, puis à partir des années 1420 le cuivre et l'argent des mines voisines de Schwaz), ainsi qu'un pouvoir dépassant lui aussi largement les limites de la région grâce à leur proximité avec les comtes de Tyrol (qui résident dans l'Innsbruck voisine), surtout à partir du moment où ceux-ci sont des Habsbourg (1379), ce qui va ouvrir grande la porte à des fonctions au niveau de l'Empire. Cette richesse extrême, eu égard aux dimensions limitées de la ville (Hans Fieger der Mittlere, à sa mort en 1503, laisse un patrimoine de 200000 florins), ainsi que l'organisation oligarchique du pouvoir municipal qui en découle (dans le dernier quart du XVe s., les quatre maires sont renouvelés tous les ans jusqu'à leur décès), amènent K.B. à rejeter la pertinence, pour l'étude des villes de la fin du Moyen Âge, de la taille de la population comme critère pour l'étude de la différenciation 
sociale ou pour celle de l'organisation politique, puisque dans ces domaines Hall montre des caractéristiques généralement considérées comme réservées aux métropoles. L'explication qu'il donne de cette non-pertinence est la suivante : l'ampleur des fortunes (et la restriction de l'accès au pouvoir politique qui en découle) ne dépend que de la fonction assurée par une ville dans un réseau urbain, les fonctions commerciales supra-régionales assurant seules la constitution de patrimoines importants ; or ces fonctions n'ont rien à voir avec le volume de la population urbaine, mais sont en rapport avec l'insertion dans les échanges à grande distance. À cet égard, Hall, située au point de rupture de charge sur l'Inn, joue, avec ses foires, un rôle pivot dans les échanges entre l'Italie et l'Allemagne, rôle renforcé par la maîtrise de biens rares (sel, métaux).

2 Un résultat important, donc. On regrettera toutefois qu'il soit obtenu à travers une méthodologie souvent défaillante : K.B. ne cesse de poser à ses sources des questions auxquelles elles ne peuvent répondre (ce qui nous vaut maints développements assez généraux exclusivement tirés de la bibliographie), et au contraire laisse largement inexploitées les richesses d'une série de comptes municipaux qui commence dès 1411 et est sans lacune à partir de 1451. Enfin, on ne peut que s'étonner, dans un travail fondé sur des comptes, de l'absence de toute série de prix, ainsi que de la façon désinvolte avec laquelle sont réglés (ou plutôt tus) les problèmes monétaires. 